\title{
Complications of intravesical BCG therapy in non-muscle invasive bladder cancer: our tertiary care centre experience
}

\author{
Vivek Sharma ${ }^{1}$, Avinash P. S. Thakur²*10, Vasantharaja Ramasamy ${ }^{3}$, Pushpendra Kumar Shukla ${ }^{4}$, \\ Fanindra Singh Solanki ${ }^{5}$, Arpan Choudhary ${ }^{2}$ and Prashant Patel ${ }^{2}$
}

\begin{abstract}
Background: Urothelial bladder carcinoma accounts for around 3.9\% cases of all the male cancers in India. Nonmuscle-invasive bladder carcinoma (NMIBC) is predominant group which constitute approximately three fourth of the urothelial bladder cancer. Intravesical BCG immunotherapy is the corner stone of today's NMIBC management. However, as with any other therapy it has its own complications and its interruption due to these adverse effects is a major cause of suboptimal efficacy. The aim of this study was to assess the complications of intravesical BCG therapy and their management in NMIBC patients.
\end{abstract}

Methods: This was a retrospective descriptive study conducted between October 2016 and November 2019; a backward review of 149 patients with diagnosis of NMIBC that undergone intravesicle BCG therapy was performed. Patient's demographical, clinical, diagnostic and procedural data regarding bladder tumour, BCG therapy, its complications and management were collected and analysed.

Results: Total 149 patients were analysed, comprising 116 males and 33 females. The mean age was of $57.2 \pm 6.7$ years. Total $85.23 \%$ were primary and $14.76 \%$ were recurrent tumours. Total 96 patients $(64.42 \%)$ completed the planned course, while 53 (35.57\%) interrupted. The reasons for BCG interruption includes adverse effects (15.4\%), progression of disease (6.7\%), disease refractory to BCG (4.6\%) and disease recurrence during BCG (3.3\%). Most of the adverse events occurred in first 6 months and most interruptions occurred after the induction period. Cystitis was the most common observed adverse effect seen in 39.6\% patients. Frequency, urgency, haematuria were common presentation. Radical cystectomy was the most common (16.10\%) further treatment with patients whose treatment was interrupted.

Conclusion: BCG is an indispensable therapy available for NMIBC, but it is associated with array of adverse effects and complications, which are the main reasons for poor compliance to BCG therapy. Although BCG-related complications can affect any organ in the body, potentially life-threatening systemic BCG-related infections are encountered in only $<5 \%$ of patients. There are some difficulties in diagnosis of the BCG complications because acid-fast staining, culture and PCR test are not always positive; tissue biopsies should be indicated sometimes to evaluate histopathology and presence of M. bovis. A persistently monitored multidisciplinary approach with high index of suspicion and prompt anti-TB therapy can help to derive the maximum benefits while keeping the complications at check.

Keywords: Intravesical BCG, Tuberculosis, Non-muscle-invasive bladder carcinoma, BCG toxicity, BCG cystitis

\footnotetext{
*Correspondence: drapst@gmail.com

${ }^{2}$ Department of Urology, Super Speciality Hospital, Netaji Subhash

Chandra Bose Medical College, Jabalpur, M.P., India

Full list of author information is available at the end of the article
}

\section{Background}

Bladder cancer is the most common malignancy of the urinary system and the ninth most common malignancy worldwide [1]. In India, urothelial bladder carcinoma 
accounts for around $3.9 \%$ cases of all the caners in the male population [2]. Non-muscle-invasive bladder carcinoma (NMIBC) constitutes around 75\% of newlydiagnosed urothelial bladder cancer and has a significant progression and high recurrence rate [3].

Transurethral resection of bladder tumour (TURBT) is a standard initial treatment for NMIBC [4]. However, TURBT alone treatment is not sufficient because of high recurrence rate $(50-70 \%)$ and high progression rate $(10 \%$ and $20 \%)$ of NMIBC over a period of 5 years $[5,6]$. Hence, to decrease it, intravesical therapies with chemotherapeutic agents have been recommended. Intravesical BCG is effective in decreasing the risk of progression and recurrence $[7,8]$.

European Association of Urology (EAU) stratified the patients into three different risk groups and instillation therapy recommendations are based on these risk groups [4]. According to EAU guidelines, patients with intermediate-risk tumours should receive full-dose BCG or chemotherapy instillations for 1 year and for high-risk tumours full-dose BCG treatment for 1-3 years is recommended. BCG is the main stay treatment of high risk NMIBC, which has been using since long time. Unfortunately, nearly $60-70 \%$ patients experience local or systemic side effects resulted from BCG and in about $<5 \%$ patients it can cause serious adverse complications $[4$, 9-12]. As compare to other intravesical chemotherapy, BCG side effects are more, so the treatment interruption rate is also higher (40\%) in this therapy [10].

BCG therapy toxicity commonly varies from local urinary symptoms to severe inflammatory response. Mostly NMIBC patients develop self-limited cystitis that may increase in severity with further treatments [13, 14]. These local or systemic symptoms may increase with each cycle gradually, so in order to prevent the long-term complications of immunotherapy a delay, interruption or a lower dose of BCG instillations is advised.

The adverse effects occurrence is one of the main reasons why urologists try to avoid the use of BCG. However, despite its toxicity, the potential benefits of BCG favour the use of this agent in patients who have moderate and high-risk NMIBC. Daily practice and adequate management of patients receiving BCG treatment is very important.

The aim of this study was to assess the complications of intravesical BCG therapy and their management in NMIBC patients.

\section{Methods}

This was a retrospective study of patients with NMIBC who had intravesical BCG immunotherapy. The study was performed at tertiary care centre from October 2016 to November 2019. Medical records of the patients with NMIBC who underwent BCG immunotherapy were anthologised. Total 156 patients who received BCG instillations as treatment for NMIBC were included. From these 156 patients, 7 patient's data could not be accessed so they were removed from the study. Finally, 149 patients were eligible for study analysis.

Inclusion criteria

1. Patients age less than 70 years,

2. Patients with NMIBC including carcinoma in situ (CIS), Ta and T1 stage,

3. Patients with good general condition to tolerate the BCG.

\section{Exclusion criteria}

1. Patients whose records were incomplete,

2. Immunosuppressed and immunocompromised patients,

3. Patient with total incontinence.

\section{Consort Diagram}

168 patients were assessed for eligibility

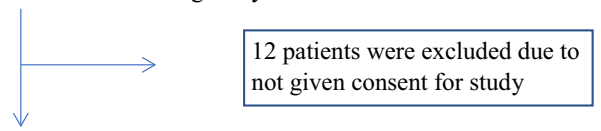

156 patients were eligible for uncontrolled, observational study

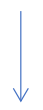

156 patients were suitable for study in initial data collection phase

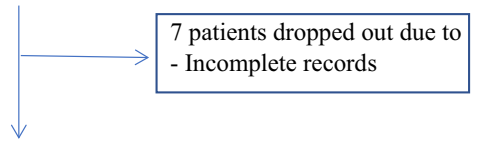

149 patient's data was analysed for final assessment

All patients were assessed by review of their records available in the department and all necessary findings were recorded.

Patient's demographical, clinical, diagnostic and procedural data regarding comorbidities, tumour size, tumour side, prior recurrence status, number of tumours, $\mathrm{T}$ category, associated CIS, tumour grade, number of TURBT performed, indication for BCG (primary or recurrent tumour), planned length of BCG maintenance therapy, total doses of BCG instillations, stopping of BCG treatment, recurrence and progression after BCG, further treatment after BCG instillations, adverse effects and complications of BCG were collected by research team and analysed. 
A TURBT operation was performed on every patient before BCG therapy. Tumours were staged and graded by TNM and WHO 2016 classifications. Residual tumours during second TURBT were categorized as the primary tumour. The second TURBT was performed in patient with incomplete initial resection, no muscle in the initial resection, all $\mathrm{T} 1$ and all high grade or G3 tumours. A biopsy-confirmed Ta, T1 tumour after 3 months and CIS after 6 months of BCG treatment were defined as recurrences during $\mathrm{BCG}$ treatment. Progression was defined as $\mathrm{pT}_{2}$ or higher disease. The planned length of $\mathrm{BCG}$ treatment was assessed according to medical records (1/2/3 years, unspecified).

\subsection{Schedule and technique of intravesical BCG instillation}

South-west Oncology Group (SWOG) regimen was followed for instillations of BCG (Moscow strain was used-itself derived Pasteur strain with each $40 \mathrm{mg}$ having 1-1.92 $\times 108 \mathrm{CFU}$ ). This regimen was consisted of an induction therapy of 6 weekly instillations after 4-6 weeks of TURBT, followed by maintenance therapy of 3 weekly instillations given at 3 and 6 months, and then every 6 months for up to 3 years. Full written informed consent was obtained from each patient before starting BCG therapy.

After excluding urinary tract infection (UTI) and haematuria, the instillation procedure was performed by urology resident in a standard manner. In case of adverse effects/complications, the decision to stop, continue or postpone treatment was taken by treating Urologist.

All patients were advised to report immediately to urology clinic if they develop symptoms of BCG adverse effect or infection.

BCG infection was confirmed via the detection of $M$. bovis by culture or M. tuberculosis complex (MAC) by polymerase chain reaction (PCR) assay or clinical, histopathologic and radiologic evidence of mycobacterial infection.

\subsection{Statistical analysis}

Data were collected by review of the records of patients. Data values were entered according to the variables onto spread sheets of Microsoft Office Excel and the variables were analysed.

For the variables which were categorical, percentage and proportions were calculated manually and the results were reported.

\section{Results}

Total 149 patients were studied, comprising of 116 $(77.85 \%)$ males and $33(22.14 \%)$ females. The mean age was of $57.2 \pm 6.7$ (range 28-70) years. Symptomatic presentation of NMIBC was seen in 132 (88.5\%) patients and diagnosis was incidental in 17 patients. History of smoking was present in 69 (46.3\%) patients. Patient's demographic features are provided in Table 1.

Tumour characteristics have been shown in Table 2 . Out of 149, 127 (85.23\%) were primary and 22 (14.76\%) were recurrent tumours. Solitary tumours were 97 $(65.1 \%)$ and multiple were 52 (34.8\%). Maximum tumours (69.79\%) were of $<3 \mathrm{~cm}$ size. Most common biopsy findings in initial TURBT were Ta high grade (33.5\%) and T1 tumour (29.5\%). After second TURBT, $34.2 \%$ patients were T0. According to EAU risk stratification 105 patients were in intermediate and 44 were in high risk category so planned BCG was 1 and 3 years, respectively. Total 96 patients completed the planned course, while 53 interrupted.

Adverse effects and complications of BCG therapy are summarized in Table 3. Cystitis was the most common observed adverse effect seen in 39 (26.2\%) patients. Frequency, urgency, haematuria were common presentation. Severe complications were also present in few patients who required interruption of BCG and treatment with antitubercular drugs (ATT).

Outcomes of the tumour and reasons for interrupting intravesical BCG are provided in Table 4. Out of 149, 96 (64.42\%) patients completed the BCG course with mild adjustment of therapy. In 53 (35.57\%) patients BCG therapy was stopped due to various reasons that includes adverse effects (23 patients; $15.4 \%$ ), progression of disease (10 patients; $6.7 \%$ ), disease refractory to BCG (7 patients; $4.6 \%$ ), disease recurrence during BCG (5 patients; $3.3 \%$ ). Most of the adverse events occurred in first 6 months and most interruptions occurred after the induction period. ATT was started in 23 (15.4\%) patients. Total 8 patients stopped BCG due to personal reasons.

\section{Table 1 Patients demographic profile}

\begin{tabular}{ll}
\hline Variable & All patients (total $\mathbf{N = 1 4 9 )}$ \\
\hline Male and female (ratio) & $116 / 33$ \\
Age in years (mean, range) & $57.2 \pm 6.7(28-70)$ \\
Symptomatic and incidental presentation & $132 / 17$ \\
Smoking history & $69 / 149(46.3 \%)$ \\
Family history & $07 / 149(4.6 \%)$ \\
Radiation history & $04 / 149(2.6 \%)$ \\
Occupation risk & $14 / 149(9.3 \%)$ \\
History of chronic UTI & $21 / 149(14.1 \%)$ \\
Comorbidities & \\
Diabetes & 22 \\
Hypertension heart disease & 31 \\
Renal insufficiency & 14 \\
\hline
\end{tabular}


Table 2 Tumour characteristics

\begin{tabular}{|c|c|}
\hline Tumour characteristics & $\begin{array}{l}\text { Number } \\
\text { of patients } \\
(N=149)\end{array}$ \\
\hline \multicolumn{2}{|l|}{ Tumour presentation } \\
\hline Primary & $127(85.23 \%)$ \\
\hline Recurrent & $22(14.76 \%)$ \\
\hline \multicolumn{2}{|l|}{ Number of the tumour } \\
\hline Single & $97(65.1 \%)$ \\
\hline Multiple & $52(34.8 \%)$ \\
\hline \multicolumn{2}{|l|}{ Size of the tumour } \\
\hline$<3 \mathrm{~cm}$ & $104(69.79 \%)$ \\
\hline$>3 \mathrm{~cm}$ & $45(30.2 \%)$ \\
\hline \multicolumn{2}{|l|}{ T stage after first TURBT } \\
\hline T0 & 11 \\
\hline Ta low grade & 19 \\
\hline Ta high grade & 50 \\
\hline $\mathrm{T} 1$ & 44 \\
\hline $\mathrm{CIS}$ & 25 \\
\hline \multicolumn{2}{|l|}{ T stage after second TURBT } \\
\hline T0 & 51 \\
\hline Ta low grade & 09 \\
\hline Ta high grade & 24 \\
\hline $\mathrm{T} 1$ & 18 \\
\hline $\mathrm{CIS}$ & 21 \\
\hline Biopsy not required & 26 \\
\hline \multicolumn{2}{|l|}{ Planned BCG course } \\
\hline 1 year & $105(70.4 \%)$ \\
\hline 3 year & $44(29.5 \%)$ \\
\hline Course completed & $96(64.4 \%)$ \\
\hline Course interrupted & $53(35.5 \%)$ \\
\hline
\end{tabular}

After a proper histologic, microbiologic and radiologic evaluation, $2(1.4 \%)$ patients fulfilled the criteria of mycobacterial infection. Radical cystectomy was the most common (24 patients; $16.10 \%$ ) further treatment for patients whose treatment was interrupted.

\section{Discussion}

Intravesical BCG is the most effective adjunctive agent and remains as the standard of care in the management of superficial bladder cancer. Although it is most effective in prevention of tumour recurrence and progression but it is also associated with significant adverse effects. The underlying pathogenic mechanisms of complications following BCG instillation remain not fully understood and on-going debate exists about whether it represents a form of hypersensitivity reaction or an active mycobacterial infection. This study aimed with bringing out the possible adverse effects associated with intravesical BCG and their management.
Table 3 Adverse effects and complications of BCG

\begin{tabular}{|c|c|}
\hline Organ or system involved & $\begin{array}{l}\text { Number } \\
\text { of the patients }\end{array}$ \\
\hline No organ affected & 62 \\
\hline \multicolumn{2}{|l|}{ Bladder } \\
\hline Cystitis & 39 \\
\hline Contracture & 2 \\
\hline \multicolumn{2}{|l|}{ Prostate } \\
\hline Granulomatous prostatitis(GP) & 5 \\
\hline Prostatic abscess & 2 \\
\hline \multicolumn{2}{|l|}{ Scrotum } \\
\hline Granulomatous epididymo orchitis & 4 \\
\hline Testicular abscess & 1 \\
\hline \multicolumn{2}{|l|}{ Upper urinary tract } \\
\hline Pyelonephritis or renal abscess & 2 \\
\hline Renal granuloma & 2 \\
\hline Penis balanitis & 2 \\
\hline \multicolumn{2}{|l|}{ Systemic } \\
\hline Fever & 6 \\
\hline Arthritis, conjunctivitis & 3 \\
\hline Granulomatous lymphadenitis & 4 \\
\hline Pulmonary granulomatous disease & 3 \\
\hline Granulomatous hepatitis & 2 \\
\hline Pneumonitis & 2 \\
\hline Salivary parotitis & 2 \\
\hline Mycotic aneurysms & 1 \\
\hline Miliary tuberculosis & 1 \\
\hline Disseminated BCG infection & 1 \\
\hline
\end{tabular}

In a study of Brausi et al. [15] (1316 patients managed with intravesical BCG), the patients reported local and systemic side effects were $62.8 \%$ and $30.6 \%$, respectively. Dysuria and mild fever are not very rare after BCG instillation. Fever after BCG therapy is not always a sign of systemic BCG infection since most fevers are limited to 24 h's duration [16]. However, patients with fever lasting beyond $24 \mathrm{~h}$ (especially if persists $>48 \mathrm{~h}$ ) or has an intermittent evening pattern are more likely to have systemic BCG infection. These patients usually require hospitalization and administration of anti-tuberculosis agents and occasionally a short period of fluoroquinolone and systemic steroids. A progressively increasing symptomatology with each BCG cycle should prompt a delay, a lower dose or interruption of BCG instillations, which may preclude long-term complications related to the immunotherapy.

The largest series of BCG complications included over 2600 patients $[16,17]$. The most common were LUTS (27-95\%), fever $(2.9 \%)$, hematuria $(1.0 \%)$, granulomatous prostatitis $(0.9 \%)$, granulomatous pneumonitis or hepatitis $(0.7 \%)$, arthralgia $(0.5 \%)$, epididymitis $(0.4 \%)$, 
Table 4 Outcomes of the tumour with intravesical BCG

\begin{tabular}{|c|c|c|}
\hline Outcome & Symptoms & $\begin{array}{l}\text { Number } \\
\text { of the patients }\end{array}$ \\
\hline Completed BCG therapy without any adverse effects & No symptoms & $42(28.2 \%)$ \\
\hline Completed BCG therapy with mild adverse effects & Mild irritative voiding symptoms, mild haematuria, fever $<38.5^{\circ} \mathrm{C}$ & $33(22.14 \%)$ \\
\hline Completed BCG therapy with dose reduction & Severe irritative voiding symptoms, haematuria, or symptoms lasting $>48 \mathrm{~h}$ & $21(14.1 \%)$ \\
\hline \multicolumn{3}{|l|}{ Stopped BCG due to } \\
\hline \multirow[t]{2}{*}{ 1. Adverse effects (moderate/severe) } & Allergic Reactions (Joint Pain, Rash) & $23(15.4 \%)$ \\
\hline & Organ Involvement (Epididymis, Liver, Lung, Kidney, Bone, Joint, Prostate) & \\
\hline 2. Disease progression & Tumour is progressing & $10(6.7 \%)$ \\
\hline 3. BCG refractory disease & Tumour is present at 3 months, CIS is present at both 3 and 6 months & $7(4.6 \%)$ \\
\hline 4. Recurrence during BCG & Tumour recurrence after initial response & $5(3.3 \%)$ \\
\hline 5. Patients' not willing for BCG continuation & & $8(5.3 \%)$ \\
\hline
\end{tabular}

and severe disseminated BCG sepsis (0.4\%), in addition very rare ureteral obstruction, rashes, renal abscesses and bladder contraction $($ all $<0.3 \%)$ [16, 17]. Here we report our experience of BCG complications.

Chemical cystitis is frequent after BCG administration which presents as irritative bladder symptoms [16]. Urinalysis and urine cultures do not show any infection generally. Symptoms normally subside in 1-2 days; however, they recur with subsequent BCG instillations. Bacterial cystitis shows infection at urinalysis/urine cultures and requires therapy with antibiotics. BCG-related cystitis appears as circumferential bladder wall thickening with urothelial enhancement [18]. In this study we found 23 cases $(15.4 \%)$ of chemical cystitis and 16 cases (10.7\%) of bacterial cystitis, which were lesser than other reported studies. Richard et al. in their study reported that abacterial cystitis and dysuria, occurring in $80 \%$ of patients, hematuria (40\%) and low-grade fever (30\%) [19]. Jacoiste et al. study found dysuria and frequency $(5-90 \%$ incidence), hematuria (1-34\% incidence) and fever (3\% incidence) [20].

Lamm et al. [16] had reported the bladder contraction rate of $0.2 \%$. Nieder et al. in their review reported it only $0.09 \%[21,22]$. In this study 2 patients $(1.3 \%)$ had bladder contractures that were managed conservatively without the need of augmentation procedure.

Granulomatous prostatitis (GP) following intravesical BCG is common due to reflux from the prostatic urethra to the prostatic ducts. Histologically there are necrotizing or non-necrotizing granulomas and acid-fast bacilli cannot be identified $[23,24]$. The majority of patients are asymptomatic and it is mostly present in peripheral zone which confuses with prostate cancer. We had 5 (3.3\%) such patients who were diagnosed with GP by the help of MRI. Stilmant described GP in case series of 6 patients receiving intravesical BCG [25]. Another study by Oates et al. showed $41 \%$ histologically proven granulomatous prostatitis after BCG [23]. In this study, 2 cases of Prostatic abscess were also seen with symptoms of fever, dysuria, and suprapubic pain. Abscess was drained by per rectal route and antibiotic coverage provided.

BCG-related epididymo-orchitis is rare (1\%) event but can occur many years after intravesical BCG therapy $[16,26]$. BCG generally affects the epididymis earlier than involving the testis. The causal mechanism is prostatic urethra-ejaculatory duct reflux. Patients present with fever, leucocytosis and scrotal swelling of acute or gradual onset. If the antibiotics fail; granulomatous epididymo-orchitis must be suspected and treated accordingly. Ultrasonography (US) shows bulky hyperaemic epididymis and/or testis, with a heterogeneous, hypoechoic, steady mass corresponding to granuloma formation [27]. A testicular mass can mimic malignancy. Rischmann et al. [28] reported the epididymitis in only $0.2 \%$ of patients in their clinical trial. Another study showed 3.5\% (10/282) patients developed epididymo orchitis with painless testicular enlargement, abscesses or cutaneous fistula [20]. We had 4 cases of post BCG epididymo-orchitis and 1 case of testicular abscess which was not responsive to antibiotics, after initial incision and drainage; orchiectomy had to be done for that abscess. Hydrocele and scrotal thickening commonly exist after BCG and we had 7 such patients.

Ureteral stricture and papillary necrosis are common urinary tract complications after BCG Therapy. Renal granuloma formation or granulomatous nephritis is a rare. Although the suspected cause is reflux, instances without documented reflux have been seen $[29,30]$. Furthermore, patients are usually asymptomatic or present with fever or mild abdominal ache. Renal granulomas are hypo vascular nodules and can be solitary, multifocal or bilateral [31, 32]. Prolonged antitubercular therapy should be initiated for granulomas, as we did in our 4 cases. Two patients developed abscess which was 
drained, no fistula formation was seen. Although spontaneous resolution of biopsy-proven renal granuloma has been reported [33], corticosteroids and antimycobacterial agents are the preferred remedies for these lesions. Nephrectomy or nephroureterectomy is the definitive remedy for patients who have non manageable fever or soreness despite the medicinal therapy [34]. We did not require nephrectomy for any of our patient. PerezJacoiste et al. [20] reported the kidney parenchymal involvement in $3.5 \%$ patients, which were manifested as nephritis (granulomatous inflammation) or renal masses on abdominal imaging. Lamm et al. [11] had reported the incidence of such renal granulomas at a rate of $0.1 \%$ ( 2 cases out of 2602), whereas we found these at a higher proportion $(2.6 \%)$ which may be attributed to Indian set up as well as to the fact that the previous study was almost 25 years back.

Balanitis is granulomatous infection of the penis, a rare complication following intravesical BCG therapy. Balanitis manifests as oedema, papules, ulceration, and abscess formation. Traumatic catheterization is a risk factor for this [35]. An Indian study reported the penile lesions (granulomatous balanitis) in 5.9\% cases after intravesical BCG [36]. This occurred in 2 patients in our study. Abscess was drained and sent for mycobacterial culture, finding infective organism of balanitis became a bit challenging as culture from the abscess were negative for usual bacteria and culture for tuberculosis came positive later on.

BCG spondylodiscitis is similar to tuberculous spondylodiscitis or Pott disease and presenting as fever, weight loss, lower back pain, leg weakness, and paraesthesia's [37, 38]. Endplate destruction of contiguous vertebral bodies with relative sparing of the disc in the early stage and a predilection for the lower thoracic spine is seen. MRI helps in identifying oedema, marrow infiltration, enhancement, spinal canal pathology and any epidural abscesses. Prevertebral and paraspinal abscesses might also choose to secondarily contain the psoas muscle mass or aorta. Some patients need abscess drainage, spinal stabilization or spinal cord decompression apart from the regular antimycobacterial therapy.

Osteomyelitis of the lengthy bones after BCG vaccination in youth has been stated, however not commonly. There are documented instances of septic arthritis of the left elbow, infections of hip and knee prostheses after intravesical BCG therapy [39, 40]. A psoas muscle abscess has been stated in the absence of vertebral involvement. In the literature up to 2018, only 23 cases of tuberculous spondylitis/vertebral osteomyelitis following intravesical BCG were reported [41]. Aljada et al. [37] reported a case of Pott's disease after BCG instillation. Van Thiel et al. [40] reported development of arthritis of the elbow eight month post-BCG therapy, a synovial aspirate grew Mycobacterium bovis and anti-tuberculous triple therapy (rifampicin, isoniazid and ethambutol) was instituted. We had 3 patients of arthritis/arthralgia. The symptoms subsided with conservative medication and discontinuation of BCG therapy. However, we did not have any patient with swelling at any joint.

Arterial pseudoaneurysm-saccular aneurysm can be seen on US or contrast-enhanced CT. Non-specific arteritis is also commonly seen. The abdominal aorta, thoracic aorta, femoral, popliteal and carotid arteries are affected in this order of decreasing frequency [42]. Additional troubles involving aorto-enteric and aorto-bronchial fistulas have been reported [32]. These patients have to be treated with open surgery and antimycobacterial therapy. Endovascular repair needs to be done but these patients who are poor candidates for surgery; associated hazard of future stent contamination should be considered [42]. Patients with pre-existing stent grafts who are on intravesical BCG treatment additionally might be at chance for future stent illness [43]. In the literature, about 30 BCG-related vascular complications have been reported [44]. In a study by Perez-Jacoiste et al. of 282 patients, mycotic aneurysms and pseudoaneurysms were seen in 16 patients (5.7\%) [20]. We had 1 such patient who was managed with intravascular stenting.

Pneumonitis can result in $1 \%$ of patients [45]. Symptoms can be fever, cough, dyspnoea and night time sweats, which are normally of gradual onset. The diagnosis can be mounted via the use of a mixture of transbronchial biopsy, bronchoalveolar lavage and imaging. Empirical therapy may also be initiated in the setting of a non-diagnostic workup. The pathogenesis of BCGrelated pneumonitis is a hypersensitivity response to disseminated BCG; this idea is seconded by finding of lymphocyte predominant bronchoalveolar lavage studies, reviews of entire healing without treatment or with corticosteroids alone and normally negative stains/cultures [46, 47]. Most patients are treated with a combination of corticosteroids and antimycobacterial agents. Chest $\mathrm{X}$-ray can also have regular findings or a diffuse reticulonodular pattern. CT images show diffuse micronodules mimicking tuberculosis, hematogenous metastases or fungal infection. Histologic findings of granulomatous contamination and diffuse alveolar damage can be seen.

As per the study by D. Lamm, the lungs complications following BCG instillation are uncommon, with an incidence of $0.3-0.7 \%$ of patients, presenting as interstitial pneumonitis or miliary dissemination $[11,20]$. We had pneumonitis in 2 patients and granulomas in 3 patients on imaging. They presented as unrelenting fever of low grade after instillation along with cough. Chest X-ray was inconclusive. HRCT showed diffuse micronodules 
in one patient and multiple patchy areas in another patient, the physician asked to stop BCG administration. These lesions then resolved with ATT administration over next six months. In comparison to western literature we found unrelenting fever to be a peculiar finding in our patients and it should alert the physicians in this regard.

Granulomatous hepatitis is rare; it can be a hypersensitivity reaction or direct infection [48]. Patients normally have fever and malaise; the liver can be enlarged and painful at physical examination. Laboratory assessment yields a peculiar hepatic panel and extended inflammatory markers. Imaging may additionally reveal hepatomegaly or ascites [49]. The diagnosis needs to be suspected clinically and tested with biopsy. Lamm [16] reported that it is manifesting in less than $1 \%$ of patients. Another study by Pérez-Jacoiste et al. [20] reported hepatitis as a manifestation in 16 cases of 281 (5.7\%). We had mild derangement of AST/ALT ratio in 14 patients and found granulomas in 2 of these patients. Granulomas when biopsied under USG guidance were positive for tuberculosis. The antitubercular agents are themselves a cause of LFT derangement and this must be kept in mind.

Granulomatous lymphadenitis following intravesical BCG has been mentioned [50]. Enlarged lymph nodes on fluorine 18 fluorodeoxyglucose study can mimic metastatic disease. Three of our patients had enlarged tender neck lymphnodes and 1 patient also had axillary lymphnodes in addition to cervical ones giving rise to suspicion of metastases. However, histopathologic findings helped in separating granulomatous inflammation as an alternative than metastatic carcinoma. The pathogenesis may moreover be energetic contamination or a hypersensitivity reaction. Anti-tubercular treatment for six months resolved the lymphadenitis completely.

Sepsis, which is reported in $1 / 15,000$ patients, is the most serious complication of BCG therapy and is possibly lethal [11]. There are no attribute imaging findings. Treatment consists of antimycobacterial combined with corticosteroids. Disseminated BCG has been reported as a rare complication, Lamm reported incidence after intravesical treatment was $0.4 \%$ [16]. We in our experience had only one unfortunate disseminated BCG infection when there was pleural effusion and bacteraemia and patient was admitted under ICU care and ventilatory support but survived with intensive therapy and later on antitubercular therapy. Mantoux test in this regard can be of great help wherein an indication can be sought of susceptibility to BCG dissemination, although it is not a very robust factor.

Other mentioned rare complications consist of peritonitis, choroiditis, parotitis and a contaminated cardiac conduction gadget [51].

\section{Dilemmas and uncertainities}

Diagnosing and treating BCG-related issues can be an arduous task. The clinical manifestations can delay or discontinue BCG instillation; nonspecific imaging findings can mimic cancers or non-mycobacterial infections. A major concern for such a patient with such signs and symptoms is whether to initiate an empirical anti-tubercular therapy or move ahead with biopsy for ascertaining diagnosis. This is based on one's personal scientific judgment and experience taking several factors along, giving due importance to severity of the complication, whether or not it is local or systemic and the invasiveness of the required biopsy procedure [51]. For mycotic pseudoaneurysm, spondylodiscitis or a reactivated infection, isolation of the infection source is preferred. Failure to pick out and treat an infection other than BCG can be disastrous. If suspicion of BCG illness is excessive and the source of the infection is not identified with use of initial cultures or histologic specimens, antimycobacterial therapy is indicated while acid-fast staining is pending.

Biopsy can be reserved for instances in which there are no response to antimycobacterial therapy. These settings include GP, renal granuloma and epididymo-orchitis. When a testicular infection proceeds to abscess formation not remitting with antibiotics, incision and drainage may be required, if necessary then orchidectomy is indicated. Similarly, for psoas abscess and renal abscess drainage may be needed.

Asymptomatic granulomatous disease places the clinician in predicament. Symptom improvement cannot be used as a marker for ample treatment. Imaging abnormalities might also additionally take time to resolve. Sometimes the frequency of the complication or history of difficult catheterization (which influences the possibility of a BCG-related complication) may be useful in guiding the decision of whether or not to take a biopsy, such as GP is common after BCG so biopsy threshold is high.

Sometimes excessive immune response can also be troublesome in these patients as the Reiter's syndrome (urethritis, arthritis, conjunctivitis) has been reported during BCG treatment; if it does, interruption of the schedule is mandatory [52].

Some limitations were present in this study. This was a non-interventional study. A direct comparison with different other intravesical therapies could have given it more inferential power. A longer follow-up also gives a better idea about long term complications of BCG.

\section{Conclusion}

BCG is an indispensable therapy available for NMIBC, but it is associated with array of adverse effects and complications, which are the main reasons for poor compliance 
to BCG therapy. Although BCG-related complications can affect any organ in the body, potentially life-threatening systemic BCG-related infections are encountered in only $<5 \%$ of patients. There are some difficulties in diagnosis of the BCG complications because acid-fast staining, culture and PCR test are not always positive; tissue biopsies should be indicated sometimes to evaluate histopathology and presence of $M$. bovis. A persistently monitored multidisciplinary approach with high index of suspicion and prompt anti-TB therapy can help to derive the maximum benefits while keeping the complications at check. Prospective, multicentre studies are necessary to precisely address the risk factors, common themes at presentation as well as optimal therapeutic approach for the complications.

\section{Abbreviations}

NMIBC: Non muscle invasive bladder cancer; TURBT: Transurethral resection of bladder tumour; BCG: Bacille Calmette-Guerin; CIS: Carcinoma in situ; UTI: Urinary tract infection; ATT: Antitubercular therapy; GP: Granulomatous prostatitis.

\section{Acknowledgements}

The authors would like to thank all co-authors who provided their clinical experience and knowledge.

\section{Authors' contributions}

APST, VS: Study concept and design. APST, VS, PKS: Data acquisition. FSS, VR: Data analysis. APST, VS: Drafting of manuscript. APST, VS, VR, FSS, PKS, AC, PP: Critical revision of the manuscript. All authors read and approved the final manuscript.

\section{Funding}

Nil.

\section{Availability of data and materials}

The datasets used and analysed during the current study are available from the corresponding author on reasonable request.

\section{Ethics approval and consent to participate}

The ethics committees name was 'Human Ethics Committee' and date of approval was 24/02/2020.

\section{Consent for publication}

Informed written consent to participate in the study was obtained from all the patients.

\section{Competing interests}

The authors declare that they have no competing interests.

\section{Author details}

${ }_{1}^{1}$ Department of Urology, Super Speciality Hospital, Shyam Shah Medical College, Rewa, M.P., India. ${ }^{2}$ Department of Urology, Super Speciality Hospital, Netaji Subhash Chandra Bose Medical College, Jabalpur, M.P., India. ${ }^{3}$ Department of Urology, Super Speciality Block, Government Medical College Thiruvananthapuram, Thiruvananthapuram, Kerala, India. ${ }^{4}$ Department of Urology, Shyam Shah Medical College, Rewa, M.P., India. ${ }^{5}$ Department of Urology, Netaji Subhash Chandra Bose Medical College, Jabalpur, M.P., India.

Received: 19 May 2020 Accepted: 27 November 2020

Published online: 09 December 2020

\section{References}

1. Ploeg M, Aben KK, Kiemeney LA (2009) The present and future burden of urinary bladder cancer in the world. World J Urol 27:289

2. Prakash G, Pal M, Odaiyappan K, Shinde R, Mishra J, Jalde D et al (2019) Bladder cancer demographics and outcome data from 2013 at a tertiary cancer hospital in India. Indian J Cancer 56:54-58

3. Burger M, Catto JW, Dalbagni G, Grossman HB, Herr H, Karakiewicz P et al (2013) Epidemiology and risk factors of urothelial bladder cancer. Eur Urol 63:234-241

4. Babjuk M, Bohle A, Burger M, Capoun O, Cohen D, Comperat EM et al (2017) EAU guidelines on non muscle-invasive urothelial carcinoma of the bladder: update 2016. Eur Urol 71:447-461

5. Chen SY, Du LD, Zhang YH (2010) Pilot study of intravesical instillation of two new generation anthracycline antibiotics in prevention of superficial bladder cancer recurrence. Chin Med J (Engl) 123:3422-3426

6. Addeo R, Caraglia M, Bellini S, Abbruzzese A, Vincenzi B, Montella L et al (2010) Randomized phase III trial on gemcitabine versus mytomicin in recurrent superficial bladder cancer: evaluation of efficacy and tolerance. J Clin Oncol 28:543-548

7. Han RF, Pan JG (2006) Can intravesical bacillus Calmette-Guerin reduce recurrence in patients with superficial bladder cancer? A metaanalysis of randomized trials. Urology 67:1216-1223

8. Shelley MD, Wilt TJ, Court J, Coles B, Kynaston H, Mason MD (2004) Intravesical bacillus Calmette-Guerin is superior to mitomycin $C$ in reducing tumour recurrence in high-risk superficial bladder cancer: a meta-analysis of randomized trials. BJU Int 93:485-490

9. Bassi P (2002) BCG (Bacillus of Calmette-Guerin) therapy of high-risk superficial bladder cancer. Surg Oncol 11:77-83

10. Saint F, Irani J, Patard JJ, Salomon L, Hoznek A, Zammattio S et al (2001) Tolerability of bacille Calmette-Guerin maintenance therapy for superficial bladder cancer. Urology 57:883-888

11. Lamm DL (2000) Efficacy and safety of Bacillus Calmette-Guérin immunotherapy in superficial bladder cancer. Clin Infect Dis 31:s86-90

12. DeHaven Jl, Traynellis C, Riggs DR, Ting E, Lamm DL (1992) Antibiotic and steroid therapy of massive systemic bacillus Calmette-Guérin toxicity. J Urol 147:738-742

13. Orihuela E, Herr HW, Pinsky CM, Whitmore WF Jr (1987) Toxicity of intravesical BCG and its management in patients with superficial bladder tumors. Cancer 60:326-333

14. Berry DL, Blumenstein BA, Magyary DL, Lamm DL, Crawford ED (1996) Local toxicity patterns associated with intravesical bacillus CalmetteGuérin: a Southwest Oncology Group Study. Int J Urol 3:98-100

15. Brausi M, Oddens J, Sylvester R, Bono A, Beek C, Andel G et al (2014) Side effects of Bacillus Calmette-Guérin (BCG) in the treatment of intermediate- and high-risk Ta, T1 papillary carcinoma of the bladder: results of the EORTC genito-urinary cancers group randomised phase 3 study comparing one-third dose with full dose and 1 year with 3 years of maintenance BCG. Eur Urol 65(1):69-76

16. Lamm DL, van der Meijden PM, Morales A, Brosman SA, Catalona WJ, Herr HW et al (1992) Incidence and treatment of complications of bacillus Calmette-Guerin intravesical therapy in superficial bladder cancer. J Urol 147(3):596-600

17. Koya MP, Simon MA, Soloway MS (2006) Complications of intravesical therapy for urothelial cancer of the bladder. J Urol 175:2004-2010

18. Steg A, Adjiman S, Debré B (1992) BCG therapy in superficial bladder tumours: complications and precautions. Eur Urol 21(suppl 2):35-40

19. Richard C, Lockyer W, Gillat DA (2001) BCG immunotherapy for superficial bladder cancer. J R Soc Med 94(6):316

20. Perez-Jacoiste AMA, Fernandez-Ruiz M, Lopez-Medrano F, Lumbreras C, Tejido A, San Juan R et al (2014) Bacillus Calmette-Guerin (BCG) infection following intravesical BCG administration as adjunctive therapy for bladder cancer: incidence, risk factors, and outcome in a single-institution series and a review of the literature. Medicine (Baltimore) 93:236-254

21. Nieder AM, Sved PD, Stein JP, Skinnerb DG, Soloway MS (2005) Cystoprostatectomy and orthotopic ileal neobladder reconstruction for management of bacille Calmette-Guérin-induced bladder contractures. Urology 65:909-912 
22. Hameed A, Sezian N, Thwaini A (2007) Bladder contracture: Review for intravesical bacillus Calmette-Guerin complication. Can J Urol 14:3745-3749

23. Oates RD, Stilmant MM, Freedlund MC, Siroky MB (1988) Granulomatous prostatitis following bacillus Calmette-Guerin immunotherapy of bladder cancer. J Urol 140(4):751-754

24. LaFontaine PD, Middleman BR, Graham SD Jr, Sanders WH (1997) Incidence of granulomatous prostatitis and acid-fast bacilli after intravesical BCG therapy. Urology 49(3):363-366

25. Stilmant $M$, Siroky MB, Johnson KB (1985) Fine needle aspiration cytology of granulomatous prostatitis induced by BCG immunotherapy of bladder cancer. Acta Cytol 29:961-966

26. Harada H, Seki M, Shinojima H, Miura M, Hirano T, Togashi M (2006) Epididymo-orchitis caused by intravesically instillated bacillus CalmetteGuérin: genetically proven using a multiplex polymerase chain reaction method. Int J Urol 13(2):183-185

27. Demers V, Pelsser V (2012) "BCGitis": a rare case of tuberculous epididymoorchitis following intravesical Bacillus Calmette-Guérin therapy. J Radiol Case Rep 6(11):16-21

28. Rischmann P, Desgrandchamps F, Malavaud B, Chopin D (2000) BCG intravesical instillations: recommendations for side-effects management. Eur Urol 37(suppl 1):33-36

29. Siskron FT 4th, Venable DD, Gonzalez E, Eastham JA (1997) Granulomatous mass in a nonrefluxing renal unit after bacillus Calmette-Guerin therapy for bladder cancer. J Urol 158(3 Pt 1):882-883

30. Mody GN, Tran V, Landman J (2009) BCGosis: case report of intravesicular bacille Calmette-Guérin causing upper tract granulomas simulating a renal mass without evidence of ipsilateral vesicoureteral reflux. Urology 73(2):444.e9-444.e10

31. Squires FB, Coakley FV, Berg WJ, Panicek DM (1999) Bilateral renal masses after local Bacillus Calmette-Guerin therapy for postcystectomy ureteral cancer. Abdom Imaging 24(2):200-201

32. Wada Y, Sugiyama Y, Kikukawa H, Kuwahara T, Takahashi W, Takamiya Tet al (2004) Isolated renal tuberculosis following intravesical Bacillus Calmette-Guérin therapy for bladder cancer. Urol Int 72(3):257-260

33. Al-Qaoud T, Brimo F, Aprikian AG, Andonian S (2015) BCG-related renal granulomas managed conservatively: a case series. Can Urol Assoc J 9(3-4):E200-E203

34. Tavolini IM, Gardiman M, Benedetto G, Bassi P (2002) Unmanageable fever and granulomatous renal mass after intracavitary upper urinary tract bacillus Calmette-Guerin therapy. J Urol 167(1):244-245

35. Yusuke H, Yoshinori H, Kenichi M, Akio H (2006) Granulomatous balanoposthitis after intravesical Bacillus-Calmette-Guerin instillation therapy. Int J Urol 13(10):1361-1363

36. Sharma V, Sethy P, Dogra P, Singh U, Das P (2011) Primary tuberculosis of glans penis after intravesical bacillus Calmette-Guerin immunotherapy. Indian J Dermatol Venereol Leprol 77(1):47-50

37. Aljada IS, Crane JK, Corriere N, Wagle DG, Amsterdam D (1999) Mycobacterium bovis BCG causing vertebral osteomyelitis (Pott's disease) following intravesical BCG therapy. J Clin Microbiol 37(6):2106-2108
38. Katz DS, Wogalter H, D'Esposito RF, Cunha BA (1992) Mycobacterium bovis vertebral osteomyelitis and psoas abscess after intravesical BCG therapy for bladder carcinoma. Urology 40(1):63-66

39. Abu-Nader R, Terrell CL (2002) Mycobacterium bovis vertebral osteomyelitis as a complication of intravesical BCG use. Mayo Clin Proc 77(4):393-397

40. Van Thiel RJ, Nossent GD, Tjon-Pian-Gi NP (1992) Articular complications of intravesical BCG treatment for bladder carcinoma. Br J Urol 70(4):446-447

41. Kusakabe T, Endo K, Nakamura I, Suzuki H, Nishimura H, Fukushima S et al (2018) Bacille Calmette-Guerin (BCG) spondylitis with adjacent mycotic aortic aneurysm after intravesical BCG therapy: a case report and literature review. BMC Infect Dis 1(18):290

42. Seastedt KP, Ahmad U, Lau C, Weigel PR, Tsang HC, Hartman BJ et al (2015) Mycotic thoracic aortic aneurysm after intravesical Bacillus Calmette-Guérin treatment. Ann Thorac Surg 99(6):2210-2212

43. Costiniuk CT, Sharapov AA, Rose GW, Veinot JP, Desjardinsc M, Brandys TM et al (2010) Mycobacterium bovis abdominal aortic and femoral artery aneurysms following intravesical bacillus Calmette-Guérin therapy for bladder cancer. Cardiovasc Pathol 19(2):e29-e32

44. Holmes BJ, LaRue RW, Black JH 3rd, Dionne K, Parrish NM, Melia MT (2014) Mycotic aortic aneurysm due to intravesical BCG immunotherapy: clinical manifestations and diagnostic challenges. Int J Mycobacteriol 3(1):60-65

45. Gupta RC, Lavengood R Jr, Smith JP (1988) Miliary tuberculosis due to intravesical bacillus Calmette-Guerin therapy. Chest 94(6):1296-1298

46. Jasmer RM, McCowin MJ, Webb WR (1996) Miliary lung disease after intravesical bacillus Calmette-Guérin immunotherapy. Radiology 201(1):43-44

47. Strich J, Brancato S, Dolan R, Maruf M, Siddiqui MR, Sanford T et al (2017) Case presentation: lung consolidation as sequelae of BCG sepsis after combined intravesical and intraurethral BCG. Urol Case Rep 13:152-153

48. Van Outryve SM, Francque SM, Gentens PA, De Pauw FF, Bogaert EV, Van Marck EA et al (2004) Bacillus Calmette-Guérin-induced granulomatous hepatitis in a patient with a superficial bladder carcinoma. Eur J Gastroenterol Hepatol 16(10):1027-1032

49. Fradet V, Gaudreau C, Perrotte P, Côté J, Paquin JM (2007) Management of hepatic granulomatous tuberculosis complicating intravesical BCG for superficial bladder cancer. Can Urol Assoc J 1(3):269-272

50. Dogan D, Zor M, Ozkisa T, Ayten O, Yigit N, Yavas I (2015) Mediastinal granulomatous lymphadenitis after intravesical bacillus Calmette-Guerin treatment mimicking distant metastasis of primary bladder carcinoma. Arch Bronconeumol 51(10):526-527

51. Green DB, Kawashima A, Menias CO, Tanaka T, Redelman-Sidi G, Bhalla S et al (2019) Complications of intravesical BCG immunotherapy for bladder cancer. RadioGraphics 39:80-94

52. Shoenfeld Y, Aron-Maor A, Tanai A, Ehrenfeld M (2001) BCG and autoimmunity: another two-edged sword. J Autoimmun 16:235-240

\section{Submit your manuscript to a SpringerOpen ${ }^{\odot}$ journal and benefit from:}

- Convenient online submission

- Rigorous peer review

- Open access: articles freely available online

- High visibility within the field

- Retaining the copyright to your article

Submit your next manuscript at springeropen.com 